\title{
Revisão sistemática evidencia ausência de estudos observacionais, objetivando o conhecimento da política nacional de plantas medicinais e fitoterápicos
}

\author{
Augusto Santana Palma Silva ${ }^{a^{*}}$ e Sheila Torres Feitosa ${ }^{b}$ \\ ${ }^{\text {a }}$ Universidade Federal do Vale do São Francisco (UNIVASF), Petrolina, PE, Brasil \\ ${ }^{\mathrm{b}}$ Universidade Norte do Paraná (UNOPAR), Juazeiro, BA, Brasil
}

Histórico do Artigo Recebido em:

15/11/2017

Aceito em:

$18 / 02 / 2018$

\section{Palavras-chave:}

Revisão sistemática; programas nacionais de saúde;

Sistema Único de Sáude.

Keywords:

Systematic review; national health programs; Unified Health System.

\begin{abstract}
RESUMO
A Política Nacional de Plantas Medicinais e Fitoterápicos (PNPMF) busca garantir o acesso seguro e o uso racional de plantas medicinais e fitoterápicos no Brasil à população usuária do Sistema Único de Saúde (SUS), representando estratégia de influência em saúde pública. Estudos mais recentes sugerem pouco interesse pela prescrição (por parte de médicos e outros profissionais) e concepções equivocadas (por parte dos usuários) acerca da eficácia e segurança desses produtos naturais, o que sugere um desconhecimento, por parte de responsáveis técnicos, usuários e gestores da saúde acerca da PNPMF, problemática norteadora do presente estudo. A revisão sistemática qualitativa foi pautada na triagem de estudos observacionais que objetivassem identificar o conhecimento da PNPMF por gestores e ou profissionais e ou usuários do SUS. A busca foi realizada em seis bancos de dados entre os dias 28 e 29 de outubro de 2017, envolvendo uso de 16 descritores combinados, utilizando-se operador booleano em três idiomas. A busca e elaboração da presente revisão obedeceram às diretrizes propostas pela recomendação PRISMA. Das 20 estratégias de busca utilizadas, não foram identificados estudos que objetivassem o conhecimento da PNPMF por usuários e ou gestores e ou profissionais do SUS. Por fim, identifica-se a necessidade de estudos envolvendo o tema em questão, ação que pode auxiliar na garantia de diretrizes da PNPMF: o estímulo à prescrição, recomendação e uso racional de plantas medicinais e fitoterápicos.
\end{abstract}

Systematic review evidences absence of observational studies objectiving the knowledge of the national policy of medicinal and phytotherapy plants

\section{ABSTRACT}

The National Policy of Medicinal Plants and Phytotherapics (PNPMF) seeks to ensure the safe access and rational use of medicinal plants and herbal medicines in Brazil to the users of the Unified Health System (SUS), representing a strategy of influence on public health. Recent studies suggest little interest in prescription (by doctors and other professionals) and misconceptions (on the part of users) about the efficacy and safety of these natural products, which suggests a lack of knowledge on the part of technical managers, users and managers about the PNPMF, which is the guiding problem of the present study. The qualitative systematic review was based on the screening of observational studies that aimed to identify the knowledge of the PNPMF by managers and / or professionals and / or SUS's users. The search was performed in six databases between October 28 and 29, 2017 involving the use of 16 descriptors combined using Boolean operators in three languages. The search and elaboration of the present revision obeyed the guidelines proposed by the PRISMA recommendation. Of the 20 search strategies used, no studies were identified that aimed at the knowledge of PNPMF by users and / or managers and / or SUS's professionals. It is identified the need for studies involving the theme in question, an action that can help to guarantee PNPMF's guidelines: the encouragement of prescription, recommendation and rational use of medicinal and phytotherapeutic plants.

\footnotetext{
* Autor correspondente: augustolestat@gmail.com (A. S. P. Silva)
} 


\section{Introdução}

A Política Nacional de Plantas Medicinais e Fitoterápicos (PNPMF), aprovada em 2006, procura garantir o acesso seguro e o uso racional de plantas medicinais e fitoterápicos no Brasil à população usuária do Sistema Único de Saúde (SUS). Representa uma política pública do SUS que busca, conforme suas diretrizes e estratégias, estimular não apenas o uso desses fitoterápicos e plantas medicinais por seus usuários, mas também a sua prescrição por profissionais da área da Saúde (1).

Apesar de configurar-se como estratégia de influência no segmento da saúde pública, estudos mais recentes apontam não apenas para um baixo interesse de profissionais da área da Saúde pela utilização de plantas medicinais e fitoterápicos (2), mas também para o fato de que parece haver concepções equivocadas acerca da eficácia e segurança destes (3), o que pode sugerir um baixo conhecimento técnico-científico dos usuários (ainda que superficial), profissionais e gestores da saúde acerca da PNPMF.

Nesse sentido, o objetivo do presente estudo foi o de sumarizar evidências relacionadas ao conhecimento, por gestores e ou usuários e ou profissionais do SUS da PNPMF.

\section{Materiais e métodos}

Trata-se de uma Revisão Sistemática qualitativa (sem meta-análise) orientada na seguinte problemática: "A Política Nacional de Plantas Medicinais e Fitoterápicos (PNPMF) é conhecida por gestores e ou pacientes e ou (futuros) profissionais da área das Ciências da Saúde envolvidos diretamente com o Sistema Único de Saúde (SUS)?". Objetivando a resposta a essa questão, uma busca sistematizada (não protocolada em função da não obrigatoriedade exigida pelo periódico) por estudos observacionais e disponíveis em sua íntegra foi realizada em bancos de dados distintos, utilizando-se 16 diferentes descritores relacionados, simultaneamente, às plantas medicinais, ao SUS e à PNPMF, em três idiomas (português, inglês e espanhol) e referentes ao período compreendido entre os anos 2006 (ano de publicação da política) e 2017 (período atual de elaboração da revisão). Esses estudos, por sua vez, deveriam ter como objetivo (principal ou secundário) o levantamento de informações - mediante quaisquer estratégias de obtenção de informações (questionário, entrevista etc.) propostas por seus respectivos autores - referente ao conhecimento, por parte de gestores e ou usuários e ou (futuros) profissionais do SUS acerca da PNPMF.

Os descritores individuais consideraram a designação proposta pelos Descritores em Saúde (DeCS) e seus respectivos sinônimos, cuja lista encontra-se abaixo na Tabela 1, que evidencia os descritores individuais, e suas respectivas relações, envolvidos na concepção das 20 estratégias de busca - cuja lista completa encontra-se ao final da presente revisão, na seção Apêndice (após as referências) e que envolvem o combinador booleano 'AND'. 
Tabela 1: Lista de descritores por relação (número sobrescrito) e respectivos idiomas utilizados na confecção das estratégias de busca envolvidas na busca de pesquisas, objetivando identificar o conhecimento, de gestores e ou usuários e ou (futuros) profissionais de saúde, acerca da Política Nacional de Plantas Medicinais e Fitoterápicos (PNPMF) do Sistema Único de Saúde (SUS).

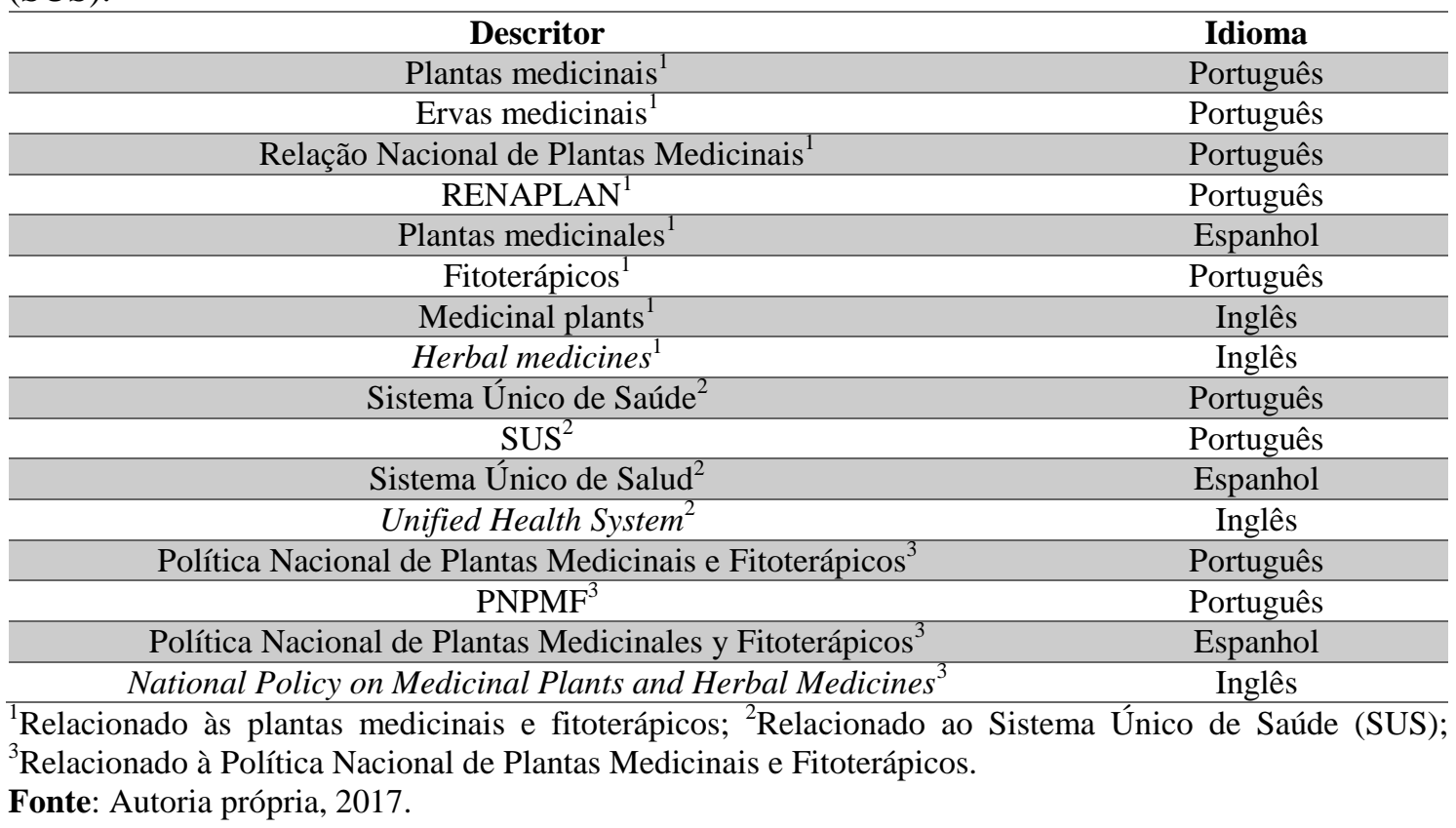

A busca por estudos potencialmente relevantes foi realizada durante os dias 28 e 29 de outubro de 2017, envolvendo seis bancos de dados ${ }^{1}$ : a) Biblioteca Virtual em Saúde (BVS); b) PubMed; c) Scientific Electronic Library Online (SciELO); d) Science Direct; e) Scopus; e f) Web of Science. A busca foi realizada utilizando computador pessoal de um dos revisores na Universidade Federal do Vale do São Francisco (UNIVASF) campus Centro - Petrolina-PE, sendo a base de dados Web of Science acessada através do Portal de Periódicos CAPES. Em cada base, os descritores envolvidos (estratégia de busca) deveriam compor seções específicas relativas a cada uma. A Tabela 2, abaixo, evidencia a data de busca, o horário de início e fim da busca e o tempo (em minutos) utilizado para a prospecção de artigos (por cada base) respectivamente envolvida(s) na triagem inicial. A Tabela 3, em seguida, discrimina as seções envolvidas na triagem por cada base consultada.

Tabela 2: Bancos de dados, data de busca, horário inicial e de término e tempo gasto (em minutos) envolvidos na triagem inicial de pesquisas com o objetivo de identificar o conhecimento, de gestores e ou usuários e ou (futuros) profissionais de saúde, acerca da Política Nacional de Plantas Medicinais e Fitoterápicos (PNPMF) do Sistema Único de Saúde (SUS).

\begin{tabular}{ccccc}
\hline Banco de dados & Data da busca & Início & Término & Tempo gasto (minutos) \\
\hline BVS $^{1}$ & $28 / 10 / 2017$ & $9: 24 \mathrm{PM}$ & $9: 43 \mathrm{PM}$ & 19 \\
\hline PubMed & $28 / 10 / 2017$ & $9: 50 \mathrm{PM}$ & $9: 59 \mathrm{PM}$ & 9 \\
\hline SciELO & $28 / 10 / 2017$ & $10: 01 \mathrm{PM}$ & $10: 07 \mathrm{PM}$ & 6 \\
\hline Science Direct & $28 / 10 / 2017$ & $10: 12 \mathrm{PM}$ & $10: 17 \mathrm{PM}$ & 5 \\
\hline Scopus & $29 / 10 / 2017$ & $2: 16 \mathrm{PM}$ & $2: 22 \mathrm{PM}$ & 6 \\
\hline Web of Science & $29 / 10 / 2017$ & $2: 30 \mathrm{PM}$ & $2: 34 \mathrm{PM}$ & 4 \\
\hline
\end{tabular}

${ }^{1}$ Biblioteca Virtual em Saúde (BVS); ${ }^{2}$ Scientifc Electronic Library Online (SciELO); *Acessado através do Portal de Periódicos CAPES.

\footnotetext{
${ }^{1}$ A seleção destas bases de dados considerou a relação da PNPMF com a área da Ciências da Saúde (BVS) bem como o fato da possibilidade de publicação em periódicos indexados PubMed, SciELO, Science Direct, Scopus e Web of Science.
} 
Tabela 3: Bancos de dados e seções envolvidas na triagem inicial de pesquisas com o objetivo de identificar o conhecimento, por parte de gestores e ou usuários e ou (futuros) profissionais de saúde, acerca da Política Nacional de Plantas Medicinais e Fitoterápicos (PNPMF) do Sistema Único de Saúde (SUS).

\begin{tabular}{|cc}
\hline \multicolumn{1}{|c}{ Banco de dados } & Seção abordada \\
\hline BVS $^{1}$ & Título, resumo, assunto \\
\hline PubMed & Todos os campos \\
\hline SciELO ${ }^{2}$ & Todos os índices \\
\hline Science Direct & All fields \\
\hline Scopus & Article title, abstract, keywords \\
\hline Web of Science* & Tópico \\
${ }^{1}$ Biblioteca Virtual em Saúde (BVS); ${ }^{2}$ Scientifc & Electronic Library Online $($ SciELO); *Acessado através \\
do Portal de Periódicos CAPES. &
\end{tabular}

Fonte: Autoria própria, 2017.

Após a identificação de resultados potencialmente relevantes, procedeu-se à identificação e exclusão de resultados em duplicata. Em seguida, à leitura de títulos e resumos no intuito de selecionar aqueles relacionados ao objetivo da presente Revisão Sistemática para compor a amostra final. Essa análise foi realizada pelos dois revisores, sendo o grau de concordância entre ambos (coeficiente Kappa) calculado. Foram desconsiderados (critérios de exclusão) artigos de revisão, estudos observacionais em desacordo ao objetivo da presente Revisão Sistemática, além de estudos não observacionais (pesquisa laboratorial), livros e estudos não realizados no Brasil. A estruturação dos resultados (tópico a seguir), bem como toda a elaboração da presente revisão, foi elaborada obedecendo-se à recomendação PRISMA para elaboração de Revisões Sistemáticas (4).

\section{Resultados e discussão}

Foram identificados, após busca nos seis bancos de dados, um total de 49 resultados potencialmente relevantes, os quais, após exclusão daqueles em duplicata (30), resultaram em uma amostra composta por 19 resultados. Após a leitura dos seus títulos e resumos, e eliminação: a) dos artigos de revisão (cinco resultados); b) dos estudos observacionais não relacionados ao objetivo da presente Revisão Sistemática (oito resultados); c) dos estudos não realizados no Brasil (dois resultados); d) dos estudos na forma de pesquisa laboratorial (três resultados); e e) livros (um resultado), não foram identificados estudos de natureza observacional que objetivassem determinar $o$ conhecimento de gestores e ou usuários e ou (futuros) profissionais do Sistema Único de Saúde (SUS) acerca da Política Nacional de Plantas Medicinais e Fitoterápicos (PNPMF). A Figura 1, abaixo, apresenta a síntese de resultados obtidos após a realização da presente Revisão Sistemática. O coeficiente Kappa obtido foi igual a 1, indicando concordância quase perfeita entre os revisores, reforçando a consistência do método utilizado no presente estudo para recuperação da informação disponível acerca do tema (5). 


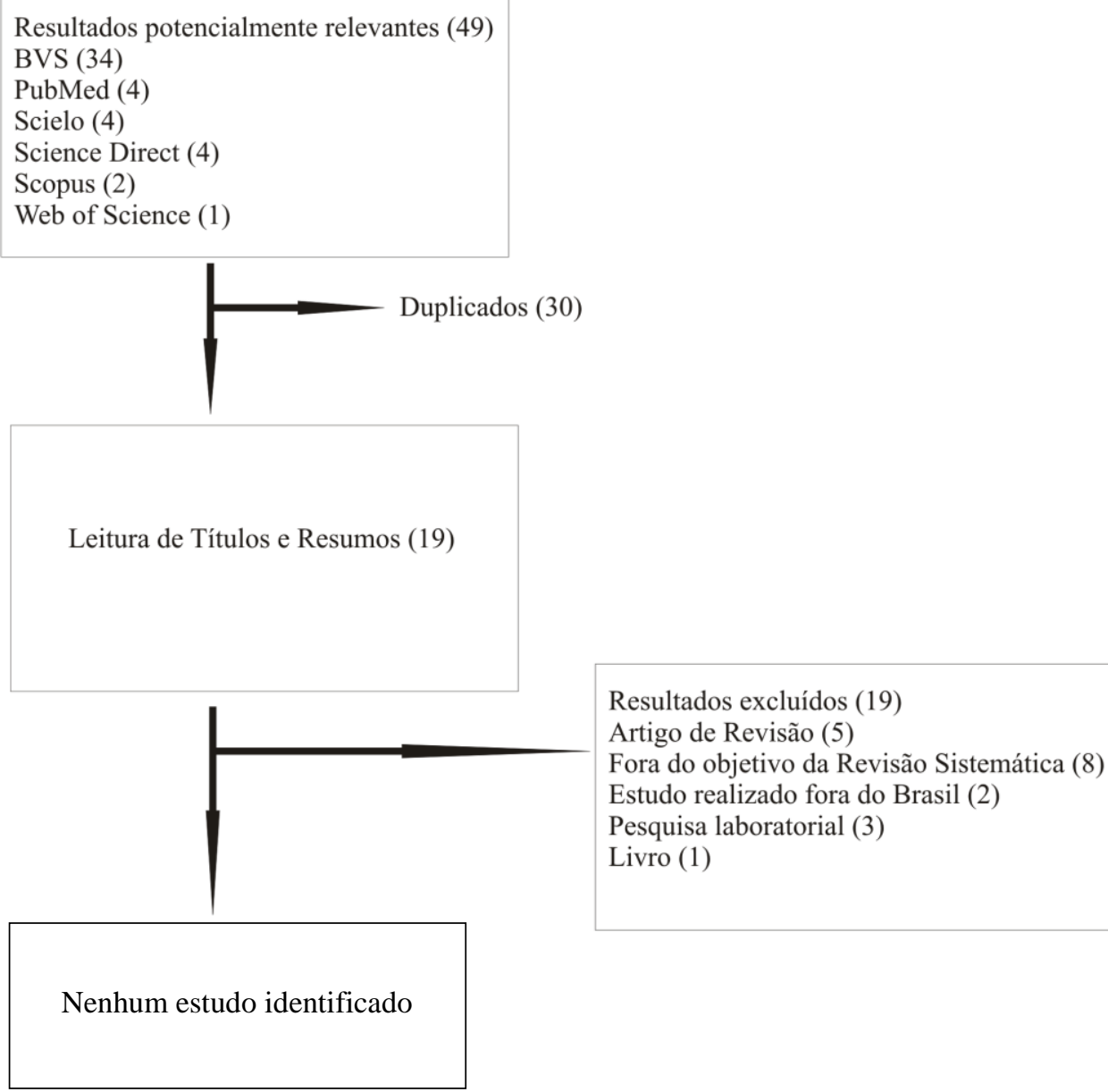

Figura 1: Diagrama de fluxo da Revisão Sistemática obtido considerando a identificação de estudos de natureza observacional cujo objetivo era identificar o conhecimento, de gestores e ou usuários e ou (futuros) profissionais de saúde acerca da Política Nacional de Plantas Medicinais e Fitoterápicos (PNPMF) do Sistema Único de Saúde (SUS).

Apesar do percentual de aproveitamento nulo para cada base consultada, observou-se um maior percentual de resultados potencialmente relevantes para a base da Biblioteca Virtual em Saúde (cujos resultados brutos foram iguais a aproximadamente 69,39\%), seguido de iguais valores percentuais $(8,16 \%)$ para as bases PubMed, SciELO e Science Direct. Finalmente, as bases Scopus e Web of Science ocuparam a terceira e a quarta posições (respectivamente) para número de resultados potencialmente relevantes $(4,08 \%$ e $2,04 \%)$. Fato semelhante foi observado em relação ao número de resultados obtidos para leitura de título e resumos após exclusão de itens duplicados, quando a mesma base (BVS) apresentou 78,95\% dos resultados (15 dos 19) que compuseram a amostra para análise, seguido da base Science Direct, com quatro dos 19 resultados (ou aproximadamente 21,05\%). As bases PubMed e Scopus continham 10,53\% (ou dois dos 19 resultados) cada e, finalmente, as bases SciELO e Web of Science apresentaram, cada, um dos resultados (ou aproximadamente 5,26\%) ${ }^{2}$. Assim, e com base no protocolo

${ }^{2}$ Ressalta-se que estes valores percentuais levam em consideração a presença de um determinado resultado em mais de uma base. 
descrito e realizado por nós, recomendamos a utilização da base de dados da BVS como estratégia de busca relevante.

Finalmente, apesar de a estratégia de Revisão Sistemática definida por nós considerar o uso de 20 distintos descritores (Apêndice) em seis bancos de dados, ressaltamos as limitações presentes em nosso protocolo, na forma da não utilização de outros bancos de dados, a exemplo da Literatura Latino-Americana e do Caribe em Ciências da Saúde (LILACS), Google Scholar, Catálogo de Teses e Dissertações da CAPES ou bancos de dados mais específicos. Além disso, o não uso de referências indiretas, bem como os próprios descritores utilizados, devem ser considerados como potencial viés para a não identificação de estudos abordando a temática.

\section{Considerações finais}

A Revisão Sistemática conduzida por nós no intuito de identificar estudos observacionais pautados na determinação do conhecimento de gestores e ou usuários e ou (futuros) profissionais da saúde da Política Nacional de Plantas Medicinais e Fitoterápicos (PNPMF) no Sistema Único de Saúde (SUS) evidenciou uma aparente ausência de estudos abordando essa temática, ausência que sinaliza uma urgente necessidade por abordagens de cunho investigativo envolvendo, principalmente, gestores e profissionais do SUS acerca do conhecimento destes da PNPMF. Adicionalmente, se considerarmos o baixo interesse de médicos e ou profissionais das Ciências da Saúde pela utilização de plantas medicinais e fitoterápicos (2), bem como o equivocado conhecimento acerca da segurança e eficácia desses por usuários do SUS (3), a presente revisão também pode orientar, como possível estratégia de implementação, ações de cunho informativo destinadas a gestores, usuários e profissionais acerca da PNPMF. Tais ações, dentre outras, caminham em direção às diretrizes da PNPMF: garantir o acesso seguro, bem como o uso racional de fitoterápicos e plantas medicinais à população brasileira, mediante a adoção de uma estratégia na forma de uma política pública (1).

\section{Referências}

1. Brasil. Ministério da Saúde. Política nacional de plantas medicinais e fitoterápicos. Brasília: Ministério da Saúde; 2006.

2. Valeriano ACFR, Junior EXS, Bedor CNG, Costa MM. O uso da fitoterapia na medicina por usuários no SUS: uma revisão sistemática. Id on Line Revista Multidisciplinar e de Psicologia 2017; 10 (33): 219-236.

3. Figueredo CA, Gurgel IGD, Junior GDG. A Política Nacional de Plantas Medicinais e Fitoterápicos: construção, perspectivas e desafios. Physis Revista de Saúde Coletiva 2014; 24 (2): 381-400.

4. Galvão TF, Pansani TSA, Harrad D. Principais itens para relatar Revisões sistemáticas e Metaanálises: A recomendação PRISMA. Epidemiologia e Serviços de Saúde, 2015; 24 (2): 335-342.

5. Kundel HL, Polansky M. Measurement of observer agreement. Radiology 2003; 228 (2): 303-308. 
APÊNDICE - Descritores utilizados como estratégia de busca nos bancos de dados

1. "plantas medicinais" AND "sistema único de saúde" AND "política nacional de plantas medicinais e fitoterápicos" AND "conhecimento"

2. "plantas medicinais" AND "SUS" AND "política nacional de plantas medicinais e fitoterápicos" AND "conhecimento"

3. "plantas medicinais" AND "sistema único de saúde" AND "PNPMF" AND "conhecimento"

4. "plantas medicinais" AND "SUS" AND "PNPMF" AND "conhecimento"

5. "ervas medicinais" AND "sistema único de saúde" AND "política nacional de plantas medicinais e fitoterápicos" AND "conhecimento"

6. "ervas medicinais" AND "SUS" AND "política nacional de plantas medicinais e fitoterápicos" AND "conhecimento"

7. "ervas medicinais" AND "sistema único de saúde" AND "PNPMF" AND "conhecimento"

8. "ervas medicinais" AND "SUS" AND "PNPMF" AND "conhecimento"

9. "relação nacional de plantas medicinais" AND "sistema único de saúde" AND "política nacional de plantas medicinais e fitoterápicos" AND "conhecimento"

10. "relação nacional de plantas medicinais" AND "SUS" AND "política nacional de plantas medicinais e fitoterápicos" AND "conhecimento"

11. "relação nacional de plantas medicinais" AND "sistema único de saúde" AND "PNPMF" AND "conhecimento"

12. "relação nacional de plantas medicinais" AND "SUS" AND "PNPMF" AND "conhecimento"

13. "RENAPLAN" AND "sistema único de saúde" AND "política nacional de plantas medicinais e fitoterápicos" AND "conhecimento"

14. "RENAPLAN" AND "SUS" AND "política nacional de plantas medicinais e fitoterápicos" AND "conhecimento"

15. "RENAPLAN" AND "sistema único de saúde" AND "PNPMF" AND "conhecimento"

16. "RENAPLAN" AND "SUS" AND "PNPMF" AND "conhecimento"

17. "plantas medicinales" AND "sistema único de salud" AND "política nacional de plantas medicinales y fitoterápicos" AND "conocimiento"

18. "fitoterápicos" AND "sistema único de salud" AND "política nacional de plantas medicinales y fitoterápicos" AND "conocimiento"

19. "medicinal plants" AND "unified health system" AND "national policy on medicinal plants and herbal medicines" AND "knowledge"

20. "herbal medicines" AND "unified health system" AND "national policy on medicinal plants and herbal medicines" AND "knowledge" 